\title{
Kayakalp: Impact of Swachh Bharat Abhiyan on cleanliness, infection control \& hygiene promotion practices in District Hospitals of Chhattisgarh, India
}

\author{
Dr Apurva Tiwari ${ }^{1}$, Ankita Tiwari $^{2}$ \\ I( National Health Mission Raipur Chhatisgarh, Kasturba Medical College/ Manipal University, India) \\ ${ }^{2}$ (National Health Mission Kawardha Chhattisgarh, School of Management/ Manipal University, India)
}

\begin{abstract}
Cleanliness \& hygiene practices in any healthcare facility are considered crucial factors while judging the quality of services rendered by them. Such Practices had always been a setback in paradigm of public healthcare. Kayakalp yojna (Award to public health care facilities), an incentivized approach was launched as a component on Swach bharat abhiyan by the prime minister of India on $2^{\text {nd }}$ October 2014, aimed at improving the Public health care facilities under six thematic areas including Hospital/ Facility Upkeep, Sanitation \& hygiene, Waste Management, Infection Control, Supportive services \&Hygiene promotion. This Study was aimed at evaluating the impact of implementation of Kayakalp Yojna across all the District Hospitals of the state of Chhattisgarh by assessing the improvement of facilities in six thematic areas. It was an analytical census study assessing all 27 District hospitals covered under this scheme during year 2015-16, source of data being public access reports available on the official website of the Department of health \& family welfare Chhattisgarh. Post implementation of Kayakalp Scheme there was an average improvement of $18.26 \%$ in the total scores of the hospital out of which Ten District hospitals showed gross improvement of more than 25\% over their initial score. Eight District Hospitals qualified for external assessment scoring above $70 \%$ on the parameters of kayakalp Guideline.
\end{abstract}

Keywords: Infection Control, Kayakalp, Peer assessment, Record review, thematic areas

\section{Introduction}

Cleanliness \& Hygiene promotion practices had always been a neglected area in purview of public health care services, demanding for deployment of strategies at its improvement. These practices affect the perception of the individual \& influence their selection of healthcare facilities (1). Once implemented it causes effective infection control within the facility leading to better heath outcome. Kayakalp yojna had evolved as one such revolutionary strategy toward up gradation of public healthcare facilities. Its main objective was to inculcate a culture for promotion of cleanliness, infection control and hygiene practices in the facilities by incentivizing \& recognizing the facilities showing exemplary performance in adhering to kayakalp guidelines \& thereafter sustaining such practices(2). The district hospitals were awarded based on their performance on six parameters. The guidelines provided with a tool for assessment of facilities thereby leading to generation of a scorecard for each facility consisting of total 500 marks, breakup of which was

1. Hospital/Facility Upkeep -100 marks

2. Sanitation and hygiene- 100 marks

3. Waste Management-100 marks

4. Infection control.- 100 marks

5. Support Services- 50 marks

6. Hygiene Promotion- 50 marks

Facilities were ranked on the basis of percentage scores obtained during the course of the assessment process. Assessment was done by using various method like observation, staff interview \&record review .(3)This whole process consisted of four steps that are, first preliminary internal assessment which was done by the team comprising of facility staff at the start of kayakalp scheme, followed by identification of gaps \& gap closure leading to final internal assessment by the same team. Thirdly facilities were then assessed by team from other district hospital under the divisional purview in form peer assessment. Those facilities obtaining a score above $70 \%$ qualified for the fourth step, the external assessment which was carried out by the team of experts assigned from state level. Based on the assessment done by the external team \& nominations hence put forward, two top ranked district hospitals received an award. The first and second best district hospital level facilities received cash award of Rs Fifty and Rs Twenty lacs respectively. Apart from these two awards all the facilities which scored above $70 \%$ in external assessment were given a motivational certificate of commendation plus a cash award of Rs three lacs each. 


\section{Methodology}

This is an analytical study done to assess the impact of Kayakalp scheme in 27 district hospitals of the state of Chhattisgarh (India) during year 2015-16, source of data being the open access reports of the census of Kayakalp scheme available on the official website of the Department of health \& family welfare Chhattisgarh.(4) A secondary data analysis of performance of the district hospitals was done under six thematic areas during various phases of assessment. Study constrain was that the data of external assessment were not available for public access; therefore the assessment of impact is based on the score of peer assessment. Parameters which were analyzed for assessment of the impact of the scheme were average percentage improvement across all district hospital, average percentage improvement in each thematic area, percentage of facilities scoring above $70 \%$, pre-post analysis of assessment of percentage improvement from the initial score at the start of the scheme. Data analysis was done using MS excel \& SPSS 20.

\section{Results \& discussion}

The Implementation of Kayakalp scheme was versed by significant level of improvement in the district hospitals of state of Chhattisgarh. Average percentage improvement in the score of district was reported to be 18.27\%.Two district hospitals i.e district hospital Jashpur \& district hospital Surguja showed significant level of improvement by more than $50 \%$ from their initial score, eight district hospital improved between 25 to 50 percent from initial score.

\section{No of District Hospitals \& Percentage improvement in scores}

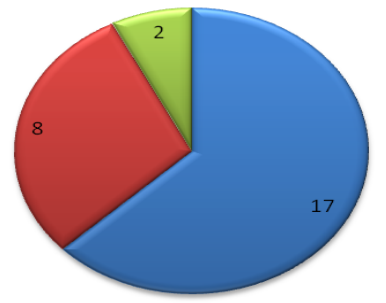

Fig.1 Percentage improvement of District Hospitals

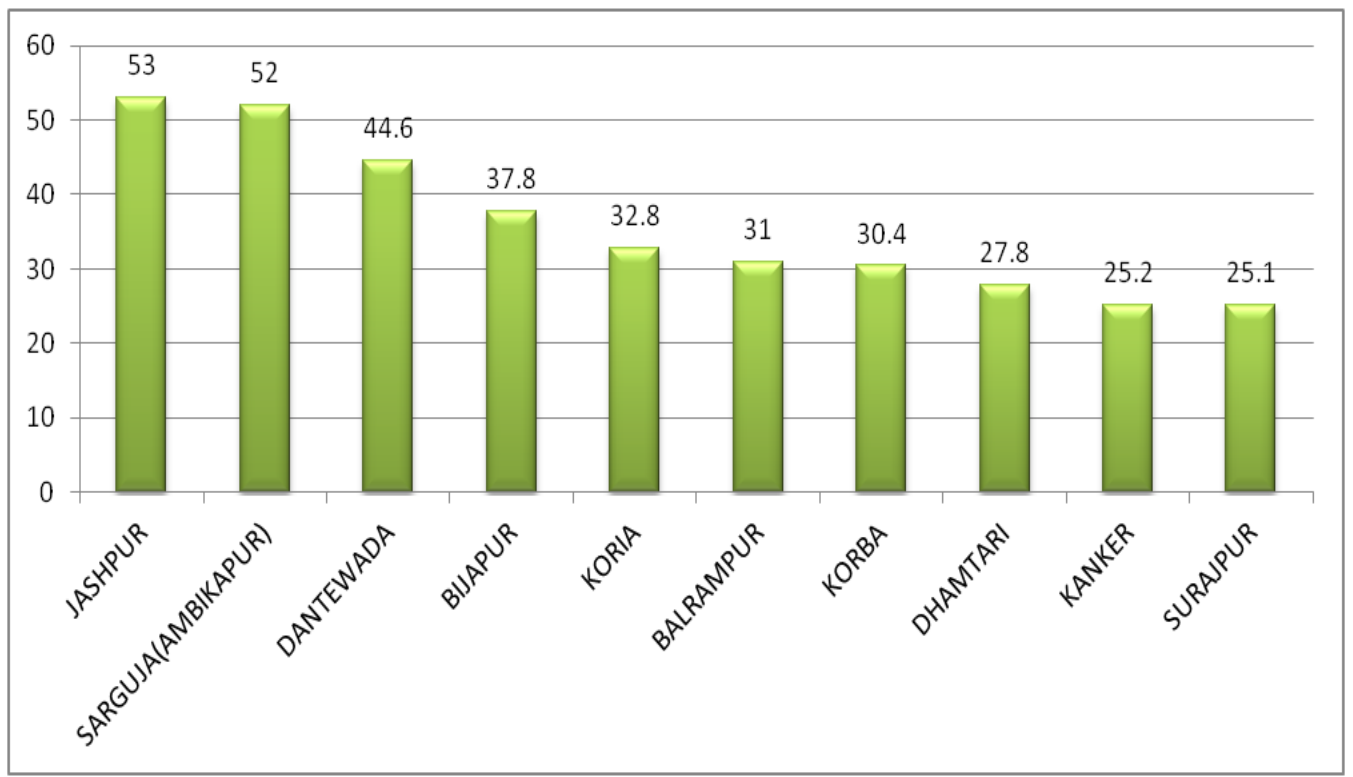

Fig2. District Hospitals with percentage improvement more than $25 \%$

Improvement in performance when analysed under six parameters of the guidelines, maximum improvement was done in the area of hygiene promotion $(23.25 \%)$ indicating toward promotion of hygiene practice like hand washing water conservation sanitation among staff \& patients visiting the facilities, followed by support services (19.91\%),Infection control(19.88\%), waste management (16\%) ,Hospital upkeep(15.87\%), sanitation \& hygiene(15.45). 


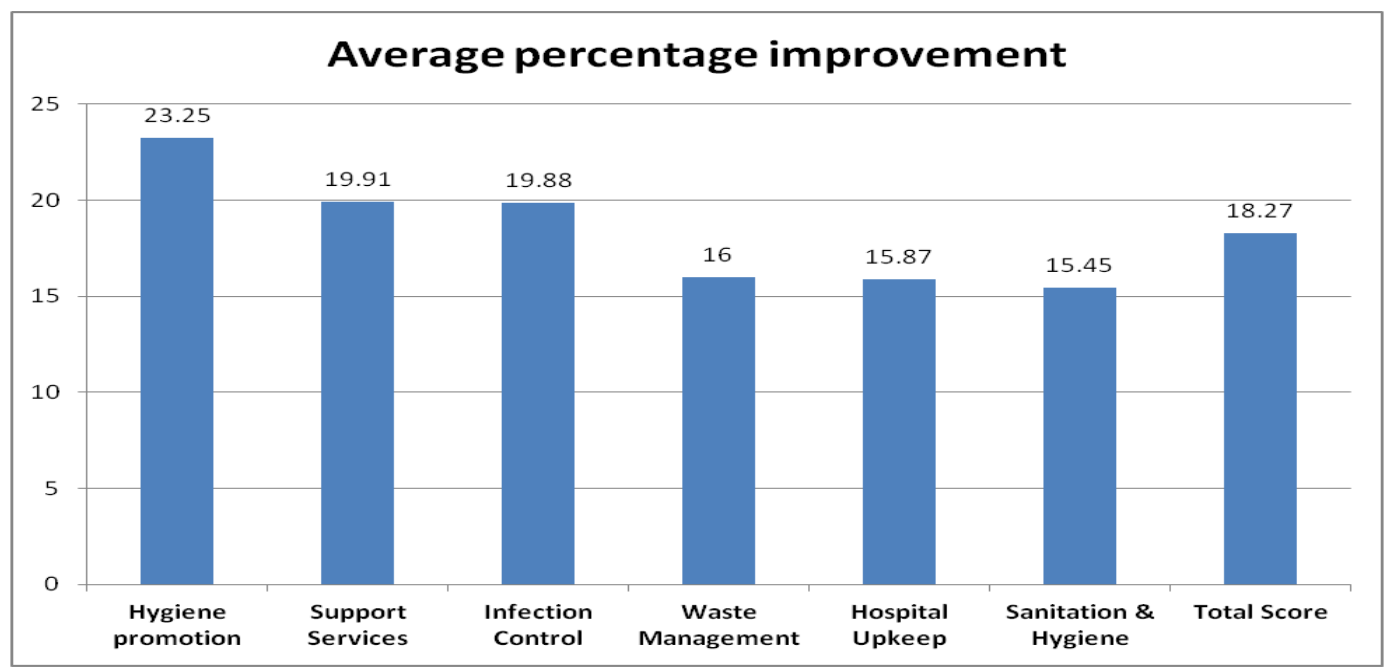

Fig3. Average percentage improvement( thematic areawise)

Among 27 District Hospitals, eight Hospitals qualified for external assessment by scoring above \& 70\% namely District Hospital Dantewada, District Hospital Jashpur, District Hospital Ambikapur, District Hospital korba, District Hospital Koriya, District Hospital Kanker, District Hospital Dhamtari \& District Hospital Bilaspur.

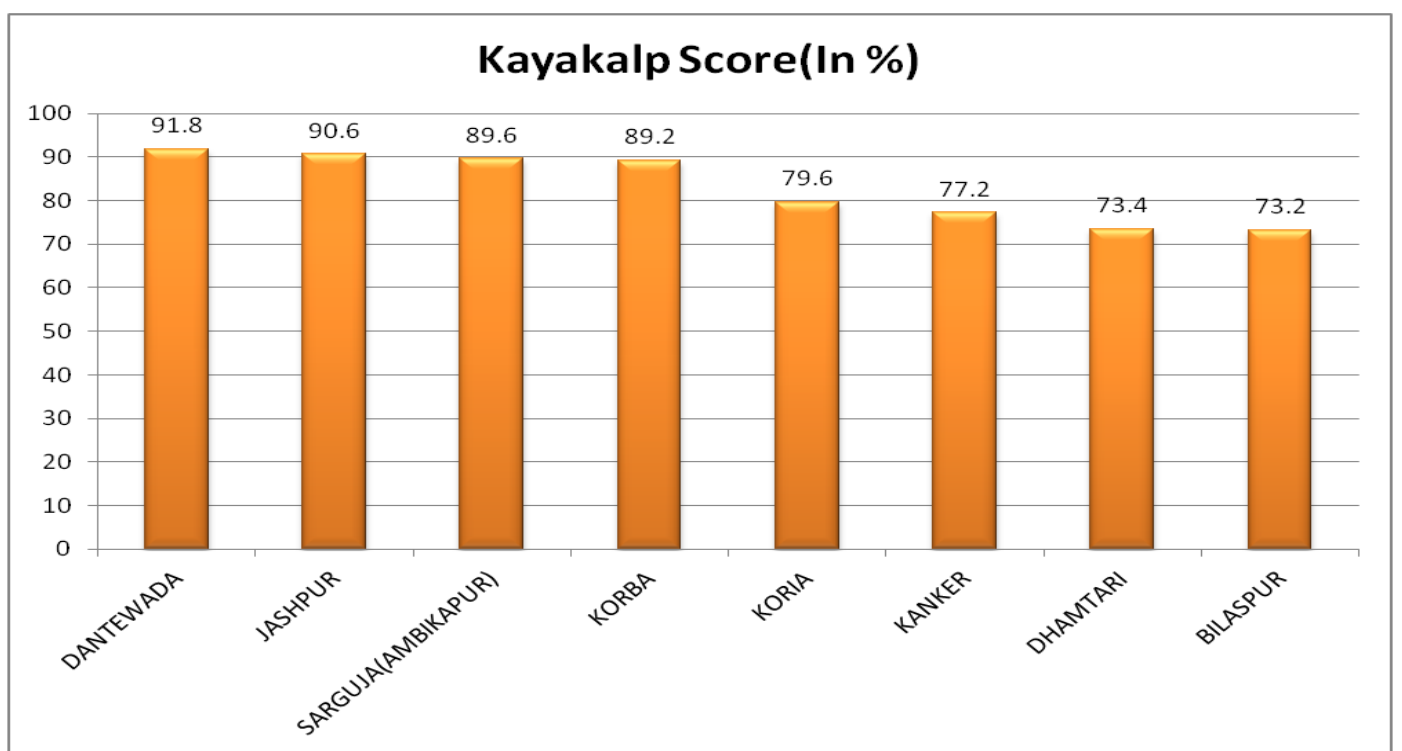

Fig4. Kayakalp peer assessment score of district Hospitals qualified for external assessment

Although the score of external assessment was not available for public access but awards were declared. First Prize was given to District Hospital Korba \& second to District Hospital Jashpur, Certificate of commendation was given to District Hospital Ambikapur, District Hospital kanker, District Hospital Dantewada \& District Hospital dhamtari.

\section{Conclusion}

Kayakalp (Award to public health care facilities), a strategy launched as a part of Swachh Bharat Abhiyan by Govt. of India proved to be a boon in up gradation of public hospitals in terms of cleanliness , hygiene \& infection Control practices in state of Chhattisgarh .Kayakalp census 2015-16 clearly highlights the impact of this incentivized approach creating a competitive clean culture in public hospitals which was once considered as a distant milestone to achieve .It not only enabled the hospitals to improve their status of cleanliness \& hygiene but an average improvement of $23.5 \%$ in hygiene promotion is indicative toward the spread of this culture to beneficiaries \& community as well. An improvement of $19.88 \%$ score in the area of infection control is supposed to reflect highly on the outcome indicators of healthcare services rendered by the facilities. 


\section{References}

[1] Aldebasi Y H and Ahmed M I (2011),Patients' Satisfaction with Medical Services in the Qassim Area, Journal of Clinical and Diagnostic Research, Vol. 5(4), . 813-817.

[2] Ministry of Health \& Family welfate govt. of India, Award to public health facilities kayakalp(Royal press, Newdelhi: Nirman Bhawan, May 2015).

[3] Ministry of Health \& Family welfate govt. of India, Swachhata guidelines for public health facilities kayakalp(Royal press, New Delhi: Nirman Bhawan, May 2015).

[4] Ministry of Health \& family welfare Chhattisgarh 2015.Kayakalp report, retrieved from http://cgweb.nic.in/health/kayakalp 